\title{
Comments on: An overview of curriculum-based course timetabling
}

\author{
Edmund K. Burke • John H. Drake • \\ Barry McCollum • Ender Özcan
}

Published online: 19 March 2015

(C) Sociedad de Estadística e Investigación Operativa 2015

Research in the area of educational timetabling has been extensive and has changed significantly over the past two decades. Timetabling and scheduling problems are amongst the most well-studied NP-hard optimisation problems due to their direct importance and relevance in real-world situations. In the early days of timetabling research, it was common for authors to present results for bespoke problem instances taken from a single institution. The introduction of standardised benchmarks for examination timetabling in 1996 (Carter et al. 1996) and for course timetabling a few years later (via the first International Timetabling Competition, ITC-2002 ${ }^{1}$ ) contributed towards creating a more cohesive research community, able to directly compare computational results obtained by different solution methods.

\footnotetext{
1 The original website for this competition no longer exists and no associated paper was produced, however the details of ITC-2002 are discussed within the description of ITC-2007 (McCollum et al. 2010).
}

This comment refers to the invited paper available at doi:10.1007/s11750-015-0366-z.

\section{E. K. Burke}

CHORDS Research Group, Computing Science and Mathematics, School of Natural Sciences, University of Stirling, Stirling FK9 4LA, UK

e-mail: e.k.burke@stir.ac.uk

\section{J. H. Drake $(\varangle) \cdot$ E. Özcan}

ASAP Research Group, School of Computer Science, University of Nottingham,

Jubilee Campus, Wollaton Road, Nottingham NG8 1BB, UK

e-mail: John.Drake@nottingham.edu.cn; drakejohnh@gmail.com

E. Özcan

e-mail: ender.ozcan@nottingham.ac.uk

B. McCollum

School of Computer Science, Queen's University Belfast, University Road,

Belfast BT7 1NN, Northern Ireland, UK

e-mail: b.mccollum@qub.ac.uk 
This overview of curriculum-based course timetabling (CB-CTT) is a well-written and informative paper. It presents an introduction to CB-CTT and it provides a comprehensive overview of the solution methods that have been used to solve this problem, with a particular focus on the instances introduced in the second International Timetabling Competition (ITC-2007) (McCollum et al. 2010).

This overview clearly highlights the state-of-the-art exact and metaheuristic methods applied to the ITC-2007 benchmark instances. The most well-known ILP formulations used for deriving upper and lower bounds are described in detail, along with a comprehensive comparison of their performance over the ITC-2007 instances. The authors note that solving such problems to optimality directly still remains a challenge, given the computational power currently available. This is directly related to an area that is not highlighted in this paper: the sharp increase in the number of parallel processing methods now being published, due mainly to the availability of affordable general purpose GPUs. Although we are not aware of any direct application of multicore or multi-threaded solutions to the ITC-2007 CB-CTT benchmarks, work does exist within educational timetabling in the context of examination timetabling (Kolonias et al. 2014) and high-school timetabling (Bożejko et al. 2014). We would suggest that this will be a growth area in timetabling research in the next few years.

In addition to the discussion of exact methods, a number of (meta)-heuristic approaches are categorised and described. One of the most significant contributions of this work is the un-biased comparison of ten state-of-the-art methods taken from the literature. A subjective analysis of performance can be made based on solution quality and the computational time used. Extensions to the CB-CTT are discussed, with reference made to fairness in timetabling, a continuing trend in most areas of timetabling and scheduling (see, for example, Ajtai et al. 1998; Martin et al. 2013a). The consideration of human factors is notoriously difficult to measure but it is becoming increasingly important when convincing people and organisations to embrace automated timetabling solutions. Fairness issues in problems such as staff scheduling (Martin et al. 2013a) and examination timetabling (Muklason et al. 2014) have a relative notion of what is considered to be 'fair' or 'unfair', with the focus very much on the impact of a solution on individuals within the system. We would argue that in the context of CB-CTT, clear definitions of fairness must be defined to move this area of research forward, taking into consideration the experiences of the stakeholders in the real-world versions of the problem. A further extension that is discussed is the idea of multi-objective CB-CTT. An overview of multi-objective meta-heuristics for timetabling problems is provided by Landa Silva et al. (2004). Multi-objective optimisation is a well-established research area with an extremely active community. Increasingly, as the questions we ask of decision support systems become more complex, the research effort aimed at multi-objective search is likely to increase. As with the issue of fairness, the relevance of 'multi-objectivity' within CB-CTT must be considered in a realistic way, drawing upon the experience of practitioners in the area.

As evidenced by the number of papers included in this overview, the benchmarks proposed in ITC-2007 have made an important contribution in maintaining an ongoing cohesive research community in educational timetabling over the past few years. In any area of search and optimisation, effective modelling of a particular problem is paramount to yielding results which are relevant in the real world. One potential 
drawback of the standardisation of timetabling benchmarks is that research is increasingly constrained by solving 'toy' problems with no practical applications. The ITC-2007 benchmarks were designed to reflect the challenges of solving real-world timetabling problems, based on existing problem instances. It is important that the emphasis on real-world practical problem solving is not lost and that the gap between theory and practice is bridged in two directions. Decision support systems developed in academia must feed back into practice, while new practical challenges observed in the real world are incorporated into academic models.

From a practitioners perspective, real-world timetabling does not solely require a good solution method. Operational issues such as data and system integration, flexibility in approach within a multi-user environment, security and access levels, data integrity, delivery and access methods are all equally important parts of an effective solution delivery. The inclusion of an easy-to-use graphical user interface (GUI) is particularly desirable as many individuals are involved with the processes associated with the construction and delivery of the institutional timetable and often have different requirements. In addition, there exist many elements of workflow which must be processed in the production of an overall solution. Anecdotally, we are aware of numerous situations within real-world timetabling where the majority of effort in constructing and managing the timetable is carried out through interfacing with the GUI. This strongly reflects the processes associated with practical timetabling, in particular the availability and indeed accuracy of the underlying data. There are challenges, therefore, around providing access to dynamic, interactive, robust scheduling algorithms that are able to aid the automation within the overall processes.

Although the authors provide references to a number of survey papers, timetabling research has propagated to such an extent that it is now difficult to cover all of the survey papers devoted to the topic. We would like to highlight the recent survey paper of Pillay (2014), dedicated to hyper-heuristic methods for educational timetabling problems. This survey paper covers all aspects of educational timetabling, including examination timetabling and high-school timetabling, in addition to university course timetabling problems. Hyper-heuristics are search methodologies which operate at a higher level of abstraction than traditional search and optimisation techniques (Burke et al. 2003). A key goal of hyper-heuristic research is to automate the heuristic design process, minimising the human intervention required to design effective problem solving methods. Hyper-heuristics have been used extensively to address educational timetabling problems with a large overlap existing between the two communities. As the power of automated search methods increases and the requirements for scheduling and timetabling applications become more sophisticated, we expect the hyper-heuristic and educational examination research communities to continue to co-evolve.

In conclusion, this paper is well motivated and well written. It represents an important contribution to the scientific literature in this area. It meets its stated objective of reviewing the mathematical models and search algorithms that have been employed to address CB-CTT. We consider this paper to be an outstanding introduction to the field for those who are unfamiliar with the area. We would like to see a new generation of researchers address the ongoing challenges of this field. Our view is that this paper will make a strong contribution to the education of newcomers to timetabling research. 


\section{References}

Ajtai M, Aspnes J, Naor M, Rabani Y, Schulman LJ, Waarts O (1998) Fairness in scheduling. J Algorithms 29(2):306-357

Bożejko W, Gniewkowski Ł, Wodecki M (2014) Solving timetabling problems on GPU. In: Rutkowski L, Korytkowski M, Scherer R, Tadeusiewicz R, Zadeh LA, Zurada JM (eds) Proceedings of 13th international conference on artificial intelligence and soft computing (ICAISC'14), vol 8468. Lecture notes in computer science. Springer, Zakopane, pp 445-455

Burke EK, Hart E, Kendall G, Newall J, Ross P, Schulenburg S (2003) Hyper-heuristics: an emerging direction in modern search technology. In: Glover F, Kochenberger G (eds) Handbook of metaheuristics, vol 16. Kluwer Academic Publishers, Dordrecht, pp 457-474

Carter MW, Laporte G, Lee SY (1996) Timetabling: algorithmic strategies and applications. J Oper Res Soc 47(3):373-383

Kolonias V, Goulas G, Gogos C, Alefragis P, Housos E (2014) Solving the examination timetabling problem in gpus. Algorithms 7(3):295-327

Landa Silva JD, Burke EK, Petrovic S (2004) An introduction to multiobjective metaheuristics for scheduling and timetabling. In: Metaheuristics for multiobjective optimisation. Lecture notes in economics and mathematical systems, vol 535, pp 91-129

Martin S, Ouelhadj D, Smet P, Berghe GV, Özcan E (2013) Cooperative search for fair nurse rosters. Expert Syst Appl 40(16):6674-6683

McCollum B, Schaerf A, Paechter B, McMullan P, Lewis R, Parkes AJ, Gaspero LD, Qu R, Burke EK (2010) Setting the research agenda in automated timetabling: the second international timetabling competition. Informs J Comput 22(1):120-130

Muklason A, Parkes AJ, McCollum B, Özcan E (2014) Fairness in examination timetabling: student preferences and extended formulations. In: Proceedings of the international conference on the practice and theory of automated timetabling (PATAT'14), New York, pp 26-29

Pillay N (2014) A review of hyper-heuristics for educational timetabling. Ann Oper Res. doi:10.1007/ s10479-014-1688-1 